\title{
Amyotrophic Lateral Sclerosis and Oral Health
}

\author{
Aakash Shah*, Purvesh Shah, Santosh Kumar Goje, Romil Shah, Bhumi Modi \\ Department of Orthodontics and Dentofacial Orthopedics, K.M. Shah Dental College and Hospital, \\ Vadodara, Gujarat, India
}

dai: https://doi.org/10.21467/ajgr.3.1.13-19

* Corresponding Author email:

aakashshah108@gmail.com

Article History

Received: 21 April 2017

Revised: 24 July 2017

Accepted: 05 August 2017

Published: 18 August 2017

Student(s)

- Aakash Shah

Academic Year: 2016-17

Course Level: Master Degree

Course Name: Master of Dental Surgery (M.D.S.)

Course year: $3^{\text {rd }}$ year

$\operatorname{Mentor}(s)$

- Purvesh Shah

- Santosh Kumar Goje

- Romil Shah

- Bhumi Modi

\begin{abstract}
Amyotrophic lateral sclerosis (ALS), commonly referred to as Lou Gehrig's disease is a progressive degenerative disorder that affects the nervous system, particularly the motor neurons. It may affect either spinal or bulbar neurons, which classify how symptoms present in the individual. Currently, the cause of ALS is unknown, although research indicates the possibility of genetic and environmental factors affecting its incidence. It is important for health care practitioners to understand amyotrophic lateral sclerosis to enable them to make modifications to patient care as needed.
\end{abstract}

Keywords: Respiratory, Amyotrophic Lateral Sclerosis, Periodontal, Oral hygiene, Pneumonia

\section{Introduction}

Amyotrophic Lateral Sclerosis (ALS), also known as Lou Gehrig's disease, is a degenerative, fatal neuromuscular disease. Each year in the United States 5600 people are newly diagnosed. There is no known cure. From date of diagnosis typical life expectancy is three to five years. During that time, the patient steadily deteriorates from a functional state into a completely paralyzed, fully dependent lifestyle. Pneumonia induced respiratory failure is the leading cause of death [1]. It is speculated that, as their disease advances, patients with ALS often find maintaining oral health quickly becomes a challenge. Recent studies associate poor oral hygiene and periodontal disease with colonized oropharyngeal respiratory pathogens that, when aspirated, may induce pneumonia [2]. Therefore, ALS patients with insufficient oral health and to an even higher degree, patients opting for mechanical ventilation are at an increased risk for developing life-threatening pneumonia. Research also supports the hypothesis that, in these susceptible patients, oral and dental health education can reduce the incidence of pneumonia [3]. As the disease progresses and oral health care responsibilities transition to the caregiver(s) dental hygiene education and instruction become pivotal to the patient's well-being. 
Amvotrobbic Lateral Sclerosis and Oral Health

As ALS patients' motor skills decline the level of difficulty in performing activities of daily living (ADL) and obtaining medical care increases. Not only is it an obstacle for the patient but, finances, transportation and treatment scheduling also complicate the matter for caregivers. Dental health care and oral hygiene, initially maintained by the patient now become the responsibility of the caregiver. Poor oral hygiene and periodontal disease have been positively linked with pneumonia, the leading cause of death in ALS patients [2]. Studies have also shown a reduction in pneumonia associated with poor oral health when preventative oral hygiene procedures are performed [2]. Therefore, it is imperative that the ALS patient, as well as their caregivers, understand not only the significance of oral health but are also trained in proper oral hygiene protocols.

\section{Significance of the Problem}

There is no current research supporting the necessity of dental/oral health treatment during the progression of ALS. Nor is there research that identifies the varying levels of oral health care required by ALS patients throughout the course of the disease. As the ALS patients' motor skills deteriorate, their ability to maintain oral health care also declines. Additionally, caregivers may not be aware of the significance of oral health in relationship to the specific needs of the patient. Oral hygiene instruction tailored to the various stages of the disease is easily disseminated; and the ALS patient's primary caregiver is in the optimal position to meet those needs. Progressive weakening of the muscles utilized for eating, breathing, drinking and swallowing creates additional obstacles for the ALS patient. Management of these symptoms may include ventilatory support. Non-invasive bi-level positive airway pressure devices (or BiPAP ${ }^{\circledR}$ ) machines are often incorporated at night for respiratory relief. Tracheostomy and direct mechanical ventilation devices are two additional treatment options, though seldom chosen by ALS patients [3]. Regardless of the decision, all these options have profound effects on the oral cavity and one's ability to maintain dental health. Because the patient's condition may rapidly change, oral hygiene education needs to be provided to both the patient and the caregiver. Obtaining dental care at a dental facility often poses its own set of complications. Not all dental practices are equipped to handle ALS patients in all of their variant stages. Patients may have to seek care from a different provider or forego dental treatment altogether. While changing health care providers can be stressful to any person, to the patient with ALS, this process may prove to be overwhelming and negatively influence their decision to seek care. Evaluation of ALS patients' dental needs is imperative in providing optimal patient care. Assessing the patient's current dental condition and oral health knowledge may provide valuable information regarding whether the inclusion of a dental component within ALS Association Multidisciplinary Clinics would be beneficial.

\subsection{Operational Definitions}

\subsubsection{Activities of Daily Living (ADL)}

Basic personal tasks in caring for oneself daily, for example, dressing, bathing and eating.

\subsubsection{Amyotrophic Lateral Sclerosis (ALS)}

It is a chronic disease which leads to degeneration of nerve cells of the central nervous system. The disorder leads to atrophy and muscle weakness. There is no known etiology for it and it is non-curable.

\subsubsection{Upper Motor Neuron}

Found in the motor cortex area of the brain, they send messages to lower motor neurons to control skeletal muscle movement. Primarily involved with maintaining muscle tone and initiation of voluntary muscle movement.

\subsubsection{Lower Motor Neuron}

Also known as anterior horn cells, they take messages from upper motor neurons to control the muscle fibers they innervate. 
Shah et al., Adv.J. Grad. Res.; Vol. 3 Issue 1, pp: 13-19, January 2018

\subsubsection{Bi-level Positive Airway Pressure (BiPAP)}

Mechanical ventilation designed to assist patients in moving air both into and out of lungs.

\subsubsection{Mechanical Ventilation}

Machines used to assist or replace spontaneous breathing.

\subsubsection{Invasive}

Artificially supported ventilation provided through a cannula or breathing tube placed directly into patient's trachea.

\subsubsection{Non-invasive}

Ventilatory assistance delivered via a nasal, oral/nasal or full-face mask designed to replicate patients' normal respiration.

\subsubsection{Ventilator-Associated Pneumonia (VAP)}

Common complication causing significant morbidity and mortality in critically ill patients, broadly defined as pneumonia developing more than 48 hours after intubation.

\subsubsection{Durable Medical Equipment (DME)}

Adaptive, reusable medical equipment used to improve patient comfort and/or prolong patient independence. Must be medically necessary and prescribed by a physician.

\section{Amyotrophic Lateral Sclerosis (ALS)}

\subsection{History}

It was Jean Martin Charcot, a French physician and founder of modern neurology who, in the 1870's, first linked the symptoms of amyotrophic lateral sclerosis to the group of nerves specifically affected by the disease [4]. It is believed Charcot employed a housemaid with ALS and was therefore able to systematically observe the clinical manifestations of her disease. His meticulous observations and descriptions of the disease remain accurate today. Throughout the world the disease is known by different names. In parts of Europe the disease is still known as Maladie de Charcot (Charcot's disease). In the United States, the disease is widely recognized as ALS or Lou Gehrig's disease; named after New York Yankees baseball legend Lou Gehrig, who retired after being diagnosed in 1939.

\subsection{Etiology}

A multitude of theories have postulated the etiology of ALS. However, to date, none have proved statistically significant. Charcot first hypothesized a correlation between poliomyelitis and the development of ALS. In 1907, Wilson's theory gained momentum through the early 1900's until research showed mercury and lead, the leading suspects, were capable of producing reversible ALS-like symptoms. Current research leans toward a vast multifactorial approach encompassing genetics, viral, autoimmune and neurotoxic hypotheses. [5]

\subsection{Clinical Features}

ALS is a disorder of the motor neurons responsible for contracting the skeletal muscles. In health, lower motor neurons, located in the brain stem and spinal cord, innervate the muscle fibers. When a motor neuron fiber becomes diseased it can no longer control the muscle it innervates and denervation occurs. Consequently, the impaired muscle fibers shrink and the muscle becomes unable to contract. If surrounding motor neurons are healthy they can regenerate nerve fibers and take over control from the denervated fibers. Despite the overall reduction in the number of motor neurons, as long as this process is maintained, muscle strength remains constant. When the rate of denervation exceeds the rate of reinnervation, muscle strength decreases. In more than half of ALS patients the progressive weakening of muscles are among the 
Amvotrobhic Lateral Sclerosis and Oral Health

first symptoms noted. Initial limb (arm or leg) weakness is more common than bulbar onset; which involves muscles controlling speech and swallowing. Typical signs of lower motor neuron degeneration include muscle weakness, muscle atrophy, muscle cramping, fasciculations, paretic bulbar palsy (dysarthria, dysphagia, sialorrhea), hyporeflexia and difficulty swallowing which can lead to life-threatening aspiration issues. Upper motor neurons are located in the brain and brain stem. They carry information from brain centers that control the muscles of the body. These neurons synapse with the lower motor neurons which transmit information to the muscles. Therefore, in order for motor commands generated in the central nervous system to reach the muscles of the body, signals must utilize both the upper and the lower motor neurons. Upper motor neuron dysfunction progresses more slowly and reveals itself in symptoms such as loss of dexterity, muscle spasticity, hyperreflexia and spastic bulbar palsy.

The type and degree of motor neuron onset are two of the main characteristics utilized in diagnosing and classifying ALS. Another popular basis used in classification is the method of acquisition. Ninety to ninetyfive percent of patients have the sporadic form of ALS (SALS); ALS with no known familial history. The remaining $5-10 \%$ have familial ALS (FALS), with a close relative having the disease. The clinical features of the two acquisition types are indistinguishable. In 1998 the El Escorial criteria was developed as the standard for classifying ALS patients in clinical research. These criteria are based on clinical evidence of lower and upper motor neuron involvement and represent the degree of diagnostic certainty.

\subsection{Diagnosis}

Currently there is no one hundred percent accurate, definitive test to diagnose ALS. Diagnosis is based on a myriad of signs, symptoms, neurological and laboratory data while simultaneously eliminating the diverse diagnostic alternatives that mimic ALS. The SOD1 genetic mutation represents the only known cause in ALS development. However, subsequent studies on American and British FALS patients, have shown that the SOD1 genetic mutation only accounts for $20 \%$ of inherited ALS [6]-[7].

\subsection{Treatment}

Predominant treatment consists of a variety of physical therapies and palliative drugs. In 1995, Rilutek ${ }^{\circledR}$ (riluzole) became the first FDA approved ALS drug. To date, it remains the only FDA approved ALS drug available in the United States. Researchers believe riluzole helps protect the motor neurons from an overproduction of glutamate; a substance involved in nervous system function which, in excess, proves toxic to the neurons [8]. Clinical trials have demonstrated a modest two to three-month extension in life expectancy [8]. Consequently, drug discovery remains a challenge, as pharmaceutical companies are unlikely to make a profit from such a small market. Hence, they do not dedicate resources to costly research and drug development. Despite these initiatives, today more than 130 years later, ALS remains an incurable disease.

\subsection{Primary Cause of Death}

Clinical death is defined as the cessation of the two-primary criteria necessary to sustain life: blood circulation and breathing. Most resources available on amyotrophic lateral sclerosis identify the leading cause of clinical death as respiratory failure [1], [9]-[11]. The pathological cause of death is diagnosed through laboratory analysis of bodily fluids and/or tissues. In the post mortem state this process is known as an autopsy. To date, very little literature exists identifying the cause of death in ALS patients through post mortem pathological analysis. It is estimated the autopsy rate among ALS patients is approximately four percent [9]. Within the body of research that does exist, the primary cause of death in more than 70 percent of the cases is either broncho-pneumonia or aspiration pneumonia [1], [9]-[11]. A twenty-two-year study following 100 ALS patients found a major discrepancy between the clinically assessed and pathologically determined cause of death [10]. A less than 20\% concordance between clinical and pathological conclusions, showed clinical assessment not to be a reliable marker of death [10]. 
Shah et al., Adv.J. Grad. Res.; Vol. 3 Issue 1, pp: 13-19, January 2018

\section{Correlation Between Oral Health and Respiratory Disease}

Recent literature supports the association between respiratory disease and oral health and confirms the oral cavity to be a harbor of infectious respiratory pathogens [2]. In 2001, following a nine-year study of 358 veterans, dental decay and the presence of cariogenic bacteria and periodontal pathogens were shown to be significant aspiration pneumonia risk factors [12]. Another study observed 189 elderly persons over a fouryear period and confirmed an association between pneumonia and decayed teeth. In this study dependence on caregivers was also linked to pneumonia [13]. A third study linked higher plaque scores with a previous history of respiratory tract infections [14]. Periodontal disease has been moderately associated with atherosclerosis, myocardial infarction and cardiovascular disease [15] and the risk of chronic obstructive pulmonary disease is also known to be significantly elevated with severe periodontal attachmentloss [16]. Pneumonia is defined as an inflammatory condition of the lung caused by bacterial, viral, fungal or parasitic infections. The risk of pneumonia is, in part, determined by the specific bacteria inhaled and the body's ability to eliminate the bacteria from the airway mucosa. To eliminate aspirated bacteria from the lower airway, multiple defense mechanisms must function properly. Poor oral hygiene and the presence of periodontal disease may foster oropharyngeal colonization of respiratory pathogens which increase the probability of aspiration pneumonia, especially in high-risk patients [2], [17]. The effectiveness of these mechanisms may also be further impaired by a variety of life conditions such as advanced age, residing in a nursing home or hospital and debilitated persons [2].

\section{Mechanical Ventilation and Respiratory Diseases}

In advanced stages of ALS the process breathing, or voluntarily exchanging oxygen and carbon dioxide, may become difficult and often results in respiratory distress. Symptoms often include headaches upon waking, physical exhaustion, labored breathing, and the inability to cough or speak long sentences. Decisions associated with ventilatory assistance become integral in ALS disease management [18]. Options include non-invasive positive pressure ventilation (NIPPV) and invasive ventilation. Both modalities demonstrate prolonged survival rates and are believed to improve the patient's overall quality of life [19][23]. Bilevel positive pressure airway machines, otherwise known as a BiPAP, are considered a non- invasive form of artificial ventilation. Unlike CPAP, which provides continuous airway pressure, the BiPAP unit provides two levels of air pressure, one on inhalation and another on expiration. Variant nasal and facial delivery systems are available to assist with oxygen intake and carbon dioxide removal. There are other forms of non-invasive assisted ventilation available. However, in ALS patients, the BiPAP system is most prevalent [24]. Noninvasive positive pressure ventilation use in ALS patients has been associated with prolonged survival rates [24]. In recent years nocturnal NIPPV use has become the treatment of choice for ALS patients suffering chronic respiratory insufficiency. The extent of NIPPV use in amyotrophic lateral sclerosis patients has not been widely studied [24]. Approximately three to five percent of ALS patients elected to have invasive ventilation [24]. Mechanical ventilatory support is provided via a tracheostomy, a surgically created opening in the trachea through which air is forced on an individualized, timed cycle. Nosocomial bacterial pneumonia, or ventilator-associated pneumonia (VAP), is the most common infection. VAP is associated with a seven to twenty-one-fold increase in the incidence of pneumonia and accounts for $47 \%$ of all ICU patient infections [25]. Another study found nosocomial pneumonia prevalence in ICU's ranged from $10 \%-65 \%$, with a thirteen to fifty-five percent fatality rate [26]. Studies conducted in the 1990's suggest ventilator- associated pneumonia is a vital outcome determinant in critically ill patients [26].

\section{Reduction of Respiratory Complications Through Oral Health Care Measures}

Poor oral health can lead to complex medical and dental issues [27]. It has also been positively linked to an increased incidence of pneumonia in ventilated, debilitated and other high-risk patient groups [2], [12]. A significant amount of research has been conducted, evincing consistent results, on the reduction of 
respiratory complications through oral hygiene measures. Standardized oral hygiene protocols can effectively decrease the colonization of dental bacteria associated with respiratory pathogens that cause pneumonia [28]-[33]. Mechanical toothbrushing and chlorhexidine mouthrinses were shown to be the two most effective modalities [28]-[33]. A 2009 study found, after implementation of an oral care protocol, a $46 \%$ reduction in ventilator-associated pneumonia cases during a twelve-month period [27]. Studies confirmed these results not only with ICU patients but also in nursing home residents [29].

The majority of ALS patients that elect to die home is variant upon geographical region. In Europe it approximately $52-63 \%$ of patients die at home in contrast to $85 \%$ electing to die at home within the United States [34]-[36]. As the disease progressed, the caregiver's burden becomes enormous; exponentially more so when mechanical ventilation is involved. Many caregivers resort to hospice or hire skilled health care workers for assistance with the constant care that is required.

\section{Conclusions}

Amyotrophic lateral sclerosis, a degenerative neural disorder, ultimately affects multiple body systems. Its poor prognosis and rapid progression lead to quick, visible symptoms. The initial symptoms vary according to the type of neural involvement in the individual. All patients afflicted with ALS show signs of dysphagia and other oral complications. The dental implications of ALS span both home and professional clinical care. As such, it is important for the dental practitioner to have an understanding of the disease and the modifications needed to treat patients with ALS.

\section{How to cite this article:}

Shah, A., Shah, P., Goje, S., Shah, R., \& Modi, B. (2017). Amyotrophic Lateral Sclerosis and Oral Health.Advanced Journal Of Graduate Research, 3(1), 13-19. doi: https://doi.org/10.21467/ajgr.3.1.13-19

\section{References}

[1] R. Spataro, M. L. Re, T. Piccoli, F. Piccoli, and V. L. Bella, "Causes and place of death in Italian patients with amyotrophic lateral sclerosis," Acta Neurologica Scandinavica, vol. 122, no. 3, pp. 217-223, 2010.

[2] F. A. Scannapieco, R. B. Bush, and S. Paju, "Associations Between Periodontal Disease and Risk for Nosocomial Bacterial Pneumonia and Chronic Obstructive Pulmonary Disease. A Systematic Review,” Annals of Periodontology, vol. 8, no. 1, pp. 54-69, 2003.

[3] J. M. Cedarbaum and N. Stambler, "Disease status and use of ventilatory support by ALS patients," Amyotrophic Lateral Sclerosis and Other Motor Neuron Disorders, vol. 2, no. 1, pp. 19-22, 2001.

[4] L. P. Rowland, "How Amyotrophic Lateral Sclerosis Got Its Name," Archives of Neurology, vol. 58, no. 3, Jan. 2001.

[5] A. D. Walling, "Amyotrophic lateral sclerosis: Lou Gehrig's disease," American family physician, vol. 59, no. 6, pp. 1489-1496, 1999.

[6] R. W. Orrell, J. J. Habgood, I. Gardiner, A. W. King, F. A. Bowe, R. A. Hallewell, S. L. Marklund, J. Greenwood, R. Lane, and J. Debelleroche, "Clinical and functional investigation of 10 missense mutations and a novel frameshift insertion mutation of the gene for copper-zinc superoxide dismutase in UK families with amyotrophic lateral sclerosis," Neurology, vol. 48, no. 3, pp. 746-751, Jan. 1997.

[7] M. E. Cudkowicz, D. Mckenna-Yasek, P. E. Sapp, W. Chin, B. Geller, D. L. Hayden, D. A. Schoenfeld, B. A. Hosler, H. R. Horvitz, and R. H. Brown, "Epidemiology of mutations in superoxide dismutase in amyotrophic lateal sclerosis," Annals of Neurology, vol. 41, no. 2, pp. 210-221, 1997.

[8] A. Cifra, F. Nani, and A. Nistri, "Riluzole is a potent drug to protect neonatal rat hypoglossal motoneurons in vitro from excitotoxicity due to glutamate uptake block," European Journal of Neuroscience, vol. 33, no. 5, pp. 899-913, 2011.

[9] K. M. Kurian, R. B. Forbes, S. Colville, and R. J. Swingler, "Cause of death and clinical grading criteria in a cohort of amyotrophic lateral sclerosis cases undergoing autopsy from the Scottish Motor Neurone Disease Register," Journal of Neurology, Neurosurgery \& Psychiatry, vol. 80, no. 1, pp. 84-87, 2008.

[10] P. Corcia, P. F. Pradat, F. Salachas, G. Bruneteau, N. L. Forestier, D. Seilhean, J. J. Hauw, and V. Meininger, "Causes of death in a post-mortem series of ALS patients," Amyotrophic Lateral Sclerosis, vol. 9, no. 1, pp. 59-62, 2008.

[11] Y. Mochizuki, A. Kawata, T. Hashimoto, H. Akiyama, H. Kawakami, T. Komori, K. Oyanagi, T. Mizutani, and S. Matsubara, "An autopsy case of familial amyotrophic lateral sclerosis withFUSR521G mutation," Amyotrophic Lateral Sclerosis and Frontotemporal Degeneration, vol. 15, no. 3-4, pp. 305-308, 2014.

[12] M. S. Terpenning, G. W. Taylor, D. E. Lopatin, C. K. Kerr, B. L. Dominguez, and W. J. Loesche, "Aspiration Pneumonia: Dental and Oral Risk Factors in an Older Veteran Population," Journal of the American Geriatrics Society, vol. 49, no. 5, pp. 557-563, 2001.

[13] S. E. Langmore, M. S. Terpenning, A. Schork, Y. Chen, J. T. Murray, D. Lopatin, and W. J. Loesche, "Predictors of Aspiration Pneumonia: How Important Is Dysphagia?,” Dysphagia, vol. 13, no. 2, pp. 69-81, 1998.

[14] P. Mojon, E. Budtz-Jorgensen, J.-P. Michel, and H. Limeback, "Oral health and history of respiratory tract infection in frail institutionalised elders," Gerodontology, vol. 14, no. 1, pp. 9-16, 1997. 
Shah et al., Adv.J. Grad. Res.; Vol. 3 Issue 1, pp: 13-19, January 2018

[15] F. A. Scannapieco, R. B. Bush, and S. Paju, "Associations Between Periodontal Disease and Risk for Atherosclerosis, Cardiovascular Disease, and Stroke. A Systematic Review," Annals of Periodontology, vol. 8, no. 1, pp. 38-53, 2003.

[16] V. Deo, M. Bhongade, S. Ansari, and R. Chavan, "Periodontitis as a potential risk factor for chronic obstructive pulmonary disease: A retrospective study,” Indian Journal of Dental Research, vol. 20, no. 4, p. 466, 2009.

[17] A. A. El-Solh, M. S. Niederman, and P. Drinka, "Nursing home-acquired pneumonia: a review of risk factors and therapeutic approaches," Current Medical Research and Opinion, vol. 26, no. 12, pp. 2707-2714, 2010.

[18] A. Radunovic, D. Annane, K. Jewitt, and N. Mustfa, "Mechanical ventilation for amyotrophic lateral sclerosis/motor neuron disease," Cochrane Database of Systematic Reviews, Jul. 2009.

[19] M. S. Sherman and H. L. Paz, "Review of Respiratory Care of the Patient with Amyotrophic Lateral Sclerosis," Respiration, vol. 61, no. 2, pp. 61-67, 2009.

[20] N. Lechtzin, J. Rothstein, L. Clawson, G. B. Diette, and C. M. Wiener, "Amyotrophic lateral sclerosis: evaluation and treatment of respiratory impairment," Amyotrophic Lateral Sclerosis and Other Motor Neuron Disorders, vol. 3, no. 1, pp. 5-13, 2002.

[21] A. H. Moss, E. A. Oppenheimer, P. Casey, P. A. Cazzolli, R. P. Roos, C. B. Stocking, and M. Siegler, "Patients with Amyotrophic Lateral Sclerosis Receiving Long-term Mechanical Ventilation,” Chest, vol. 110, no. 1, pp. 249-255, 1996.

[22] L. Ng and F. Khan, "Multidisciplinary care for adults with amyotrophic lateral sclerosis or motor neuron disease," Cochrane Database of Systematic Reviews, Jul. 2009.

[23] R. L. Vender, D. Mauger, S. Walsh, S. Alam, and Z. Simmons, "Respiratory systems abnormalities and clinical milestones for patients with amyotrophic lateral sclerosis with emphasis upon survival," Amyotrophic Lateral Sclerosis, vol. 8, no. 1, pp. 36-41, 2007.

[24] N. Lechtzin, C. M. Wiener, L. Clawson, M. C. Davidson, F. Anderson, N. Gowda, and G. B. Diette, "Use of noninvasive ventilation in patients with amyotrophic lateral sclerosis," Amyotrophic Lateral Sclerosis and Other Motor Neuron Disorders, vol. 5, no. 1, pp. 9-15, 2004.

[25] M. H. Kollef and D. P. Schuster, "Ventilator-associated pneumonia: clinical considerations.," American Journal of Roentgenology, vol. 163, no. 5, pp. 1031-1035, 1994.

[26] S. Randall and T. Alfred, "Dental management of long-term amyotrophic lateral sclerosis: Case report," Special Care in Dentistry, vol. 13, no. 6, pp. 241-244, 1993.

[27] A. Berry, P. Davidson, J. Masters, K. Rolls, and R. Ollerton, "Effects of three approaches to standardized oral hygiene to reduce bacterial colonization and ventilator associated pneumonia in mechanically ventilated patients: A randomised control trial," International Journal of Nursing Studies, vol. 48, no. 6, pp. 681-688, 2011.

[28] A. Azarpazhooh and J. L. Leake, "Systematic Review of the Association Between Respiratory Diseases and Oral Health," Journal of Periodontology, vol. 77, no. 9, pp. 1465-1482, 2006.

[29] H. Tantipong, C. Morkchareonpong, S. Jaiyindee, and V. Thamlikitkul, "Randomized Controlled Trial and Meta-analysis of Oral Decontamination with 2\% Chlorhexidine Solution for the Prevention of Ventilator-Associated Pneumonia," Infection Control \& Hospital Epidemiology, vol. 29, no. 02, pp. 131-136, 2008.

[30] R. Garcia, L. Jendresky, L. Colbert, A. Bailey, M. Zaman, and M. Majumder, "Reducing Ventilator-Associated Pneumonia Through Advanced Oral-Dental Care: A 48-Month Study,” American Journal of Critical Care, vol. 18, no. 6, pp. 523-532, 2009.

[31] E. Y. Chan, A. Ruest, M. O. Meade, and D. J. Cook, "Oral decontamination for prevention of pneumonia in mechanically ventilated adults: systematic review and meta-analysis," Bmj, vol. 334, no. 7599, pp. 889-889, 2007.

[32] F. Fourrier, E. Cau-Pottier, H. Boutigny, M. Roussel-Delvallez, M. Jourdain, and C. Chopin, "Effects of dental plaque antiseptic decontamination on bacterial colonization and nosocomial infections in critically ill patients," Intensive Care Medicine, vol. 26, no. 9, pp. 1239-1247, 2000.

[33] C. Neudert, D. Oliver, M. Wasner, and G. D. Borasio, "The course of the terminal phase in patients with amyotrophic lateral sclerosis," Journal of Neurology, vol. 248, no. 7, pp. 612-616, Jan. 2001.

[34] N. Lechtzin, C. M. Wiener, L. Clawson, V. Chaudhry, and G. B. Diette, "Hospitalization in amyotrophic lateral sclerosis: Causes, costs, and outcomes," Neurology, vol. 56, no. 6, pp. 753-757, 2001.

[35] L. Ganzini, W. S. Johnston, and M. J. Silveira, "The final month of life in patients with ALS," Neurology, vol. 59, no. 3, pp. 428431, 2002.

[36] J. Gil, B. Funalot, A. Verschueren, V. Danel-Brunaud, W. Camu, N. Vandenberghe, C. Desnuelle, N. Guy, J. P. Camdessanche, P. Cintas, L. Carluer, S. Pittion, G. Nicolas, P. Corcia, M.-C. Fleury, C. Maugras, G. Besson, G. L. Masson, and P. Couratier, "Causes of death amongst French patients with amyotrophic lateral sclerosis: a prospective study," European Journal of Neurology, vol. 15, no. 11 , pp. $1245-1251,2008$

\section{Publish your research article in AIJR journals-}

$\checkmark \quad$ Online Submission and Tracking

$\checkmark$ Peer Reviewed

$\checkmark$ Rapid decision

$\checkmark$ Immediate Publication after acceptance

$\checkmark$ Open Access (Articles freely available online)

$\checkmark$ Retain full copyright of your article.

Submit your article at journals.aijr.in 